Elsevier Editorial System(tm) for Design Studies

Manuscript Draft

Manuscript Number: DESTUD-D-08-00074R1

Title: Transforming shape in design: Observations from studies of sketching

Article Type: Full Length Article

Keywords: drawings, conceptual design, shape transformations, shape rules, computer-aided design

Corresponding Author: Mr miquel prats, Ph.D.

Corresponding Author's Institution: The Open University

First Author: Miquel Prats, Ph.D.

Order of Authors: Miquel Prats, Ph.D.; Sungwoo Lim, Ph.D.; lestyn Jowers, Ph.D.; Steve W Garner, Ph.D; Scott Chase, Ph.D.

Response to Reviewers: See attached document 


\title{
Transforming shape in design: Observations from studies of sketching
}

\begin{abstract}
This paper is concerned with how design shapes are generated and explored by means of sketching. It presents research into the way designers transform shapes from one state to another using sketch representations. An experimental investigation of the sketching processes of designers is presented. Connections between sketches are defined in terms of shape transformations and described according to shape rules. These rules provide a formal description of the shape exploration process and develop understanding of the mechanics of sketching in design. The paper concludes by discussing the important phenomenon of 'subshape' and suggests that a computational mechanism for detecting sub-shapes in design sketches might augment explorative sketching by providing important opportunities for manipulating and generating shape in design.
\end{abstract}

Keywords: drawings, conceptual design, shape transformations, shape rules, computer-aided design

In creative design, free-hand sketches are frequently used to record ideas for later use and to rapidly explore design alternatives. A key benefit of sketching, in fields such as product design and architectural design, is that it assists designers in the development of various characteristics of design ideas such as form and shape in a low-cost, fast and flexible way. Studies of design suggest that designers proceed by cycles of see-move-see (Schön and Wiggins 1992). Seeing concerns a process of reinterpretation of design elements in a sketch and moving concerns transformations of the reinterpreted design elements. This two-way conversation between designer and representation - the maker and the made - commonly results in the generation of sequences of related sketches in which design elements are repeated, recognised and manipulated (Prats and Earl 2006). Some research has focused on the patterns and associations that designers see in their sketches (Liu 1995, Suwa and Tversky 1997), and it has been suggested that such patterns are valuable externalisations of designers' cognitive processes (Lawson 2006). However there has been little research into the mechanisms used to transform design elements in sketches. Our research goes some way toward this goal.

The challenge of developing computational tools to support sketching in the early stages of design has produced significant interest in the design research community (Saund and Moran, 1994, Gross and Do 1996, Leclercq 1999). CAD (Computer-aided design) tools have proven to be highly effective for supporting down-stream design tasks such as analysis or manufacture and it is believed that they could also be valuable in the exploration of design alternatives, possibly by suggesting new paths of exploration to designers (Woodbury and Burrow 2006). However, the mechanisms necessary to fluidly explore design alternatives are not readily 
afforded by current computational design tools. As noted above, reinterpretation of conceptual representations is a vital aspect of design exploration and CAD has traditionally struggled to support this because of the precise and structured geometry through which it works. There can be an inconsistency between patterns and structures that are perceived in a CAD model and the transformations that are afforded by the formal geometric structures used to construct the model (Scrivener 1982). In order for CAD systems to support the early stages of design this inconsistency needs to be resolved. In particular it is necessary to explore mechanisms that can assist designers in recognising and transforming perceived design structures, regardless of method of construction.

This paper primarily outlines an experimental investigation into the sketching of architects and industrial designers. The investigation was particularly concerned with the developing form of design concepts, and the shape transformations that led to this development. Analysis of the data produced led to the definition of a set of shape transformations commonly used in sketching. The transformations are formalised according to shape rules that explicitly describe connections between sketches and allow for a better understanding of sketching in design. We also include speculation on how this knowledge might assist the development of a computerbased mechanism that might scrutinise designers' sketch outputs and lead to interactive and automatic manipulation, and perhaps even the generation of shape conjecture. It is proposed that the key to this development lies in understanding 'sub-shapes' manipulated in sequences of design sketches.

\section{Sequence of sketches}

Sketching is a common activity in a range of design disciplines. Although the styles of sketches may differ between different disciplines, the role that sketching plays is broadly similar in the creation, development, evaluation, communication and sharing of ideas (Garner 1990). Schön and Wiggins (1992) suggest that designers in the early stages of creation use two distinct interactive processes when exploring sketches, namely seeing and moving. For example, using pen and paper, a designer can quickly produce a series of sketches. Looking at these the designer may see patterns, discover associations or spot opportunities, and these perceptions can inspire further sketching where the reinterpreted forms are manipulated to inform emerging design concepts. This interactive process leads to the generation of a range of related sketches where, to a greater or lesser extent, each design concept is related to other concepts that were generated within the same exploratory process. Such related sketches have been referred to as 'design families' (Prats and Garner 2006).

In this paper, sketches are analysed based on their geometric properties. However, sketches are more than a grouping of abstract shapes. They represent ideas such as buildings or products. Thus, a circle drawn in a sketch can represent innumerable things depending on the context and what the designer has in mind. The meanings attached to shapes in a sketch can be ambiguous and dynamic, changing as a result of new interpretations of the sketch or design problem (Purcell 1998). For example, these changes can result from a geometric reinterpretation of the shapes in a sketch, or from a functional reinterpretation. Over the years, there has been much research in developing computational systems that recognise meaning in sketched shapes, and if the context is known then such systems can be used successfully to symbolically recognise specific design elements (Leclercq 1999). However, the development of 
context independent systems that can recognise what sketches represent and make contributions to the process of design exploration is problematic. One step to realising such systems involves understanding how shapes, as geometric objects, are manipulated in sketches independently of their meaning. This understanding could lead to computational systems that provide affordances that are more suitable for creative design than is currently available in CAD systems.

Figure 1 shows a much-publicised example of sketching as a means for exploring design concepts. These sketches were produced by Philippe Starck when designing the well-known Juicy Salif lemon squeezer. A picture of the final design is shown on the left, and a sequence of sketches produced during the design process is illustrated on the right. Lloyd et al. (2003) suggest that the sketches follow an anti-clockwise path from the right, evolving from a conventional lemon squeezer into a three-legged lemon squeezer. Observing sketches such as these, it is possible to perceive similarities in their shape and form. While the source of these similarities might be hard to identify, the existence of the similarities suggests that the process of developing designs might trace logical paths from an original idea to a final design. It is likely that Stark did not develop the Juicy Salif concept merely by playing with shapes. Even so, it may be possible that, after explicitly describing the successive shape transformations from sketches, new paths of exploration could be proposed by combining the described shape transformations in new ways. Such propositions could enhance the creative processes of designers by exposing them to previously unimagined opportunities for exploration (Woodbury and Burrow 2006).

Figure 1. 'Juicy Salif' (copyright Alessi), and early sketches generated by Starck to explore lemon squeezers

\section{Shape transformation and shape rules}

Shape transformations in sketches have been studied as a way of understanding a design process. Goel (1995), for example, identified lateral and vertical transformations in sketches. Lateral transformations convert one idea into a different idea, while vertical transformations manipulate one idea into a version of the same idea. Goel's research suggests that designers do not generate independent ideas but instead they generate a single idea or a few related ideas and develop them through transformations. Do et al. (2000) examined a collection of sketches made by an architect during a project. They developed a coding scheme to classify state transformations of design elements from one sketch to another. The results show that once the architect positioned an element, both the design and its context could be elaborated and reformulated. They conclude that the transformations applied by the architect are simple but in combination the process is complex. Similarly, Bilda and Demirkan (2003) captured the sketching activity of six interior designers by using a coding scheme. Their results suggest that the media in which the sketches are made - whether in paper or by computer - effects the conception of designs, one of the reasons being that digital media does not support manipulation of reinterpreted shapes. To some extent the research reported here is an 
extension of these experimental studies in the sense that they examine shape transformations in sketches as a means to inform the development of future CAD systems.

To develop the discussion it is useful to consider more formal procedures of transformation in design; to introduce the notions of shape rules and shape grammars and particularly to establish the phenomenon of sub-shapes. Knuth (1975) suggests that expressing knowledge formally can lead to a better understanding. In the context of this paper formal means computable, structured, and rigorous, not ad hoc, and for designers this means being better equipped to manipulate ideas, reflect on them, and transmit them more effectively (Abelson and Sussman 1990). For example, English grammar formally captures the principles of English language through a set of linguistic rules. These rules assist in the composition of new sentences that can be analysed and interpreted by others. Similarly, shape rules (Stiny and Gips 1972) provide a means for describing shape and shape transformations in a formal and visual way. Here, a shape is defined as a finite arrangement of geometric elements, such as points, lines, and planes, each with a definite boundary and finite, but non-zero extent. Similarly, any arrangement of geometric elements that can be perceived to be embedded in a shape is defined to be a sub-shape. Clearly any pictorial representation of a design, such as a sketch, can be formally represented as a shape, and any design elements that can be perceived to be a feature of the design can be formally represented as sub-shapes (Stiny 1990). Consequently, analysing the sketching process in terms of shape rules may assist in developing an understanding of the fundamental principles of design generation and exploration. In the remainder of this paper the terms 'design element' and 'sub-shape' will be used interchangeably, depending on context.

A shape rule is a replacement rule of the form $A \rightarrow B$, where $A$ and $B$ are both shapes. Figure $2 a$ gives an example shape rule which rotates a square 45 degrees around its centre. A rule is applicable to a shape $S$ (e.g. Figure $2 b$ ) if the shape on the left-hand side of the rule is a subshape of $S$, under a similarity transformation. For example, if a square is found to be a subshape of shape $S$ then it can be rotated 45 degrees. Figure $2 c$ shows three different examples of applying the rule in Figure $2 a$ to the shape in Figure $2 b$.

Figure 2. a) A shape rule, b) a shape $S$, and c) examples of applying the shape rule to the shape $S$

Shape grammars are defined according to sets of rules, and an initial shape to which these rules can be successively applied. They have been used as a means for analysing designs in professions such as architecture (Koning and Eizenberg 1981) and product design (Agarwal and Cagan 1998). These grammars do not seek to describe the path traced by the designer when the original works were conceived. Instead they attempt to explain design works by formalising the spatial relations between their elements. In addition, these grammars also seek to generate design alternatives that share the same design space with those designs analysed. In contrast to these previous works, this paper uses the concept of shape rules to explicitly describe shape transformations between sketches. In other words, it attempts to formally describe the path traced by designers with the aim to gain an understanding of how shapes and sub-shapes are manipulated whilst exploring design alternatives. 
Shape rules can be generalised according to schemas, as described by Stiny (2006). These rule schemas can be very useful in the study of shape transformation in design exploration since they facilitate an expression of such transformations in a formal way, but without being too specific about the detail. In this paper, the notion of schemas is used to express this more informal shape transformation process where shape rules are not meant to describe exact transformations of any given shape.

In order to formally describe shape transformations in sketches in a way that reflects the path followed by the designer more information is required than can be provided by merely viewing the sketches. In this experiment we utilised interviews with designers and video records of designers undertaking set tasks to assist the description of shape transformations into shape rules.

\section{Experimental design}

The experiment consisted of a series of design tasks that involved sketching. Eight professional industrial designers, four professional architects and two researchers in the field of architecture participated in this study. All participants had more than 3 years of professional experience in their respective disciplines.

\subsection{The tasks}

A design representation such as a sketch can be perceived in many different ways. Each interpretation leads to a decomposition of the shape into elements with relations among elements which yields a starting point for exploring variations through the generative description (Stiny, 2006). Based on this sequence we chose to run three tasks as measures for three concepts that are particularly significant in the process of shape transformation in explorative sketching - shape decomposition, shape reinterpretation, and design families.

The first task was intended to examine whether stroke-sequence whilst sketching is related to interpretation and decomposition, and was based on the work of Van Sommers (1984). The outcomes of this introductory task, however, fall outside of the scope of this paper and will not be discussed here.

The goal of the second task was to encourage a variety of shape interpretations leading to different sub-shape compositions. In order to achieve this, the participants were presented with an initial design proposal which is rich in suggestion - namely a triquetra (Figure 3). The design brief the participants were asked to respond to was dependent on their design discipline. The industrial designers were introduced to the triquetra as a concept design for a lemon squeezer, while the architects were introduced to the same shape as a conceptual design for a building. The analysis here sought to examine the extent to which a given meaning led to different paths of exploration and types of shape transformations. Participants had 10 minutes for this task.

The third task provided a more explicit initial design in order to keep participants centered on one particular design proposal and thus produce larger design families. This intentionally offered less freedom in interpretation than the second task and here two alternative shapes were given; a concept for a kettle design (for the industrial designers) and a new building (for architects). Participants had 15 minutes for this task. 
In both tasks participants were told that their first sketch should be a copy of the given starting concept as a common reference point and after that they were free to explore their own variations of the design concept.

Figure 3. Task 2: Triquetra, and Task 3: a Kettle and St Mary Axe Building

\subsection{Method}

The design tasks performed by the participants were recorded on video. While sketching, designers made use of an A4 paper-based digital notepad and this gave the dual advantage of resembling a traditional pencil-and-paper environment whilst facilitating the recording of pen stokes via screen capture software. Figure 4 shows an example of the two video streams - from video camera and video screen capture - synchronised in order to accurately interpret participants' movements and gestures whilst sketching.

Figure 4. Two synchronised videos. Left, sketches taken from the digital notepad and right, video recording of hand movements

The analysis exploited all three outputs: the sketches on paper, the video data, and the sketches recorded via the digital notepad. The sketches on paper proved useful when examining the drawings in fine detail, e.g. exploring pen pressure. The video allowed close observation of hand movements when sketching - sometimes participants made gestures suggesting a shape but without actually drawing anything. Sketches recorded via the digital notepad assisted researchers in the examination of sequence of strokes and sequence of sketches, as illustrated in Figure 5. Here, the arrows indicate the sequence in which the participant developed the sketches. This study examined transformations between successive sketches. Thus when participants returned to a previous sketch in order to further modify it (e.g. min 01:52 and min 03:35 in Figure 5) then the resulting modified sketch was considered as a new entry in the sketching sequence.

Figure 5. Stroke-by-stroke sequence recorded via a digital notepad

The recording of the sequence of strokes was particularly crucial for the analysis of task 1 (not discussed in this paper) and was also valuable in tasks 2 and 3 to track the sequence in which sketches were produced. For example, Figure 5 illustrates stroke-by-stroke how the sketch is constructed and how the participant returned to a previous sketch to add more detail. At the end of the experiment participants were shown their sketches and asked to report their design movements. Figure 6 shows three examples. 
Figure 6. Left, the hand hides elements that are not part of the first idea. Centre, hands emulate how the structure of the design was changed. Right, the participant indicates two designs that represent the same idea

\subsection{Analysis process}

Participants produced a total of just under 300 sketches and these were categorised by tasks for each participant. In our analysis the sketches were analysed as abstract shapes, without regard to function or meaning. Note that, whilst we do not argue that function and meaning are not important in the design exploration process, we do argue that for the purposes of this paper these could be neglected. This is because this work is concerned with investigating the mechanics of sketching, e.g. the shape transformations carried out by designers as they explore, rather than the motivations for these transformations. During analysis, the sketches were first ordered according to the sequence in which they were produced and then clustered for each individual designer into design families - groups of designs that represent variations of the same idea.

Figure 7 illustrates some examples of sketches from different design families used in this study. Each sequence of sketches includes a label that identifies the number of task and number of participant (ID for industrial designers and Arch for architects). Each individual sketch is also labeled with a letter and a number. The letters identify which design families sketches belong to and the numbers indicate the number in the sequence in which the sketches were produced. Note that there is no relation between letters and numbers from different participants and different tasks. Interviews in particular greatly assisted the process of identifying design families. 'I returned here because I rejected this one' or 'here we could say that I was saturated... then I thought let's go back to this initial idea' are some examples of transcripts taken from interviews that assisted in detecting design families.

Figure 7. Some sketches from different participants and different tasks that illustrate different design families

Once the sketches were arranged and clustered into design families, shape transformations between subsequent sketches were identified and described in the form of shape rules. For example, Figure 8 shows the three shape rules that were defined to describe the transformations observed between sketch 9 and sketch 10 in the top row of Figure 7 . Rule 1 stretches the pinnacle of the squeezer vertically and horizontally, rule 2 removes a line from the base, and rule 3 rounds a corner of the base. These rules describe very specific shape transformations and as a result numerous rules were needed to describe all the shape manipulations used by each participant in each task - e.g. 16 rules were needed to describe transformations in design family A (task 2 - ID6). The use of shape rules at this stage of the analysis assisted the task of describing shape transformations. Firstly, because they explicitly capture the elements recognised by the researcher and secondly, because many shape transformations are difficult to describe by text, but can be simply described in terms of shape.

Figure 8. Examples of specific shape rules. Rule 1 - outline transformation of the pinnacle, rule 2 - delete line, and rule 3 - outline transformation of the base 
In order to develop a higher-level understanding of the shape transformations represented in these specific rules, they were grouped into general shape rule types and rule schemas. This task was carried out separately by two researchers; one responsible for analysing the outputs of industrial designers and the other focusing on the architects. Interestingly, the results of the analysis of the two researchers were comparable, and they produced similar groupings of shape rules, resulting in sets of general rules, as illustrated in Figure 9. The major difference between the analyses of the two researchers arises as a result of adoption of different levels of generality - the general rules that resulted from analysing the architects' data were at a lower level than the rules arising from analysing the industrial designers' data. Consideration of both sets of general rules resulted in a common set of seven generalised shape transformation rules. The next section introduces these general rules.

\section{General shape transformations}

It is proposed that shape transformations applied to successive sketches can be described via a limited number of general shape rules. While the general rules presented here may be sufficient to capture the shape transformations of these particular participants the set of rules is not assumed to be complete. It is possible that further experimentation might result in additions to the set. Nevertheless, the general rules are: Outline transformation, Structure transformation, Substitute element, Add element, Delete element, Cut element, and Change view. Figure 9 illustrates this set of rules (left column) along with rule schemas that express each type of shape transformation in an abstract way (central column), and examples of shape transformation taken from sketches produced by participants (right column).

- Outline transformation: This rule describes (i) transformations on the path between two points forming a curved or straight line, and (ii) transformations of the length or width of an element. A shape transformation is regarded as Outline transformation if at least one end point of the transformed element is retained. Otherwise, such transformation may be considered as a change in position of the element (that is, a Structure transformation). Outline transformations primarily seem to be used for shape refinements.

- Structure transformation: This rule describes (i) translation, rotation, reflection and scale of elements, and (ii) splitting of an element into two or more elements, for example, splitting a circle into two crescents. Structure transformations are mainly used to explore different spatial relations between elements. Because Structure transformations tend to affect the layout of a design they are more likely to lead to radical changes than Outline transformations.

- Substitute element: This rule describes the replacement of an existing element in a sketch with a new element. A shape transformation is regarded as Substitute element only if the added shape is new, meaning that it has not been achieved through any other type of transformation described in the presented list.

- Add element: This rule describes the introduction of an element to a sketch. A transformation is regarded as Add element when a sketch displays an element that is not present in the preceding sketch in the sequence. 
- Delete element: This rule describes the removal of an element from a sketch. As opposed to Add element, Delete element occurs when an element is missed out from a sketch - or struck through on reconsideration of an earlier sketch - but is present in the preceding sketch.

- Cut element: This rule describes a division made to an element. In contrast to Structure transformation, Cut element occurs only if one of the parts is kept and the rest is removed.

- Change view: This rule describes a change of the viewpoint of the design. A transformation is regarded as Change view if, for example, one sketch is represented in side view and the following sketch is represented in perspective view. In contrast to the other types of transformation in the list Change view does not represent a shape transformation of the design. However, even if two sketches represent the same design, Change view normally leads to two geometrically different shapes, as for example in the case of a cylinder which can be represented by a rectangle or a circle, depending on the viewpoint.

Figure 9. General shape rules

Most sketches modifications exhibit more than one shape transformation at a time. The rules in Figure 8 show an example in which three shape transformations are used to describe the transition from one sketch to the next one. Our experiments can not reveal the exact process designers followed when transforming their sketches but participants' explanations such as "...then what I did was to bring one petal up, bring another down, and the other a bit towards here, and I came up with this" greatly assisted in the examination of transformations, especially when supported by recorded hand gestures.

\section{Testing the general rules: Observation of sketches}

Task 2 was specifically designed to promote shape reinterpretation. Accordingly, more instances of reinterpretation were found in task 2 than in task 3 in which the given initial proposal was less ambiguous. For example, seven participants in total had more than one interpretation of the initial proposal during the second task. No instances of reinterpretation of the initial proposal were detected in task 3 . These results support the claims that ambiguity triggers reinterpretation (Goel 1995). In addition, our study shows that in most cases reinterpretation leads to different sub-shapes compositions. For example, participant ID 3 initially interpreted the triquetra as a horizontal section of the pinnacle of the lemon squeezer, and then he interpreted the triquetra as a side view of the device. Later, he used the same shape as top view. Figure 10 illustrates the different sub-shapes detected in the initial shape by ID 3 during task 2. The sub-shapes were identified according to sequences of strokes - as proposed by Van Sommers (1984) - and participants' pointing gestures during interview where there was a general tendency of using their fingers or pens to follow the contour of the elements while talking about them. 
Figure 10. a) interpreted as a horizontal section of the pinnacle composed of one single element, b) side view of the lemon squeezer composed of two elements (pinnacle and base), and c) top view composed of four elements (the pinnacle in the centre and three legs around it)

Both tasks 2 and 3 suggest that designers explore design concepts within design families. In general, design families can be identified by asking participants to describe the connections between sketches. At this stage of the analysis, the contribution of the participant is essential to avoid superficial and erroneous grouping of design ideas based solely on researchers' perception. In our analysis we found that in some cases two apparently different designs were reported to be variants of the same idea and, conversely, two designs that exhibit similar characteristics were reported as to be originated from different design ideas. For example, observing the three kettles in Figure 7 (Task 3 - ID 5) one may situate the three sketches in the same design family because they contain the same elements - base, body, spout, lid and handle - in the same structural position. It is also possible to place sketch 4 in a different design family since the body of the kettle is circular, unlike the body of the kettles in sketches 6 and 8 . However, the designer claimed that sketch 4 and 6 belong to the same family while sketch 8 belongs in a different family. The participant pointed out that the shape of the body in sketch 6 was as a result of inverting the two curves that define the circular body in sketch 4 . In regard to sketch 8 , the participant commented that he started from nothing and was influenced by the petal shapes used in task 2 .

In contrast to design families, shape transformations were more challenging to identify because during the interview process participants felt more comfortable describing the connections between sketches and interpretation of their drawings than describing in detail the shape transformations they applied between their sketches. Instead the transformations identified were largely based on the interpretation of the researchers who were conducting the analysis. As a result, there is significant subjectivity in the transformations used to formalise the sketching processes of the participants. In order to examine the degree of this subjectivity two additional researchers were asked to analyse the transformations within five design families. Each researcher described the transformations of 40 sketch transitions and their results were then compared with the original analysis of the sketches.

As expected, there were considerable differences among the analyses of the researchers. In most cases the analyses did not concur because either one of the researchers did not consider some slight detail or because of different styles of coding. For example, one researcher considered that an element was deleted because it is not in the next sketch. The other researcher considered that the element was simply not in the sketch because the designer was attending to a different element and did not include other elements in order to save time. In this case, the discrepancy between the two researchers' analyses resulted from one researcher considering only the marks on the paper, while the other considered the explorative strategy of the designer. This suggests that some training previous to the analysis might ensure that the approach used is consistent for all researchers. However, further discrepancies that arose involving different interpretations of the sub-shapes manipulated by the designers could not be addressed in this way. Figure 11 shows an example of such a discrepancy between the researchers. Two researchers interpreted that the resulting sketch (Figure 11, top right) was achieved by modifying the spatial relation between three petals. Another researcher interpreted that the designer deleted one of the petals in the transformation process. Thus, the researchers 
perceived that the resulting sketch was composed of different sub-shapes - either of three petals, or of two overlapping petals.

Figure 11. Interpretation $A$ shows the rules used by two coders. Interpretation $B$ shows the rules used by another coder

\section{Discussion}

This study has shown that given a limited number of shape rules it is possible to explicitly describe shape transformations between successive sketches. Shape rules offer a means to capture information not embedded in sketches which might be useful for the recording, reflection, and reuse of previous ideas. The set of rules presented in this paper is not exhaustive and more general rules may be added. Certainly, additional rules to describe more accurate shape transformations can also be added to the set. Such rules should be regarded as an extension of general rules and therefore remain at a lower level in a hierarchy of shape transformations (Lim et al. 2008).

The analysis of the sketches produced by the design subjects has allowed the identification of sub-shapes and their transformations. These appear to be key graphic devices for design exploration. It appears that the explorative strategies of designers are tuned to look for such sub-shapes and their recognition, repetition and modification mark important iterations in creative shape transformation and development. Another conclusion that can be drawn from this study is that it is not straightforward to accurately make conjecture regarding designers' shape transformations, largely because shapes and sub-shapes can be interpreted in many different ways, and Figure 11 showed an example of this. Nonetheless, this research attempted to introduce a preliminary framework to assist the examination of the mechanisms used by designers in recognising and transforming perceived design structures. If such mechanisms could be realised it could also be possible to develop a more independent system that scrutinises designers' freehand drawing, seeking to offer potentially valuable transformations. Figure 12 illustrates a hypothetical example that shows that given an initial concept and a set of rules representative of a designer's explorative strategy, then a system computing shapes can automatically generate alternatives that could be useful to designers. These alternatives are achieved by application of the set of rules to different sub-shapes found in the design.

Figure 12. Diversity of design outcomes with various combinations of shape rules that are applied for an initial design

If this parsing of the sketching process could take place in real time it might provide a useful stimulus to the creative generation and development of shape and form. In order to develop such mechanisms it is necessary to consider not only the technical issues of recognising and manipulating perceived structures in a design but also the modes in which they could be implemented in a computational system intended to support design exploration (McKay et al. 2008). Such modes should take into account the mechanisms of shape transformation commonly utilised by designers when sketching so that they may be able to use them in ways that are cognitively comfortable (Cross 2006). 
Our vision for such a system is seen as three intertwined cycles as illustrated in Figure 13. The designer designing shapes and the system computing shapes are independent of each other and joined by a third cycle of communication between the two. Information flowing from the designer to the computer is envisioned to be in the form of commonly used design descriptions, such as sketches and in the form of shape rules. Information flowing back to the designer will be in the form of lattices of computed shapes that prompt and inspire the designer. The communication cycle is critical in order to ensure a fluid interaction between designer and computer. The research reported here aims to provide insights that enable a closing of this communicating cycle. Our future research plans are to involve designers in the process of describing their shape transformations with rules, and investigate potential benefits of computational systems that support explorative processes through generation of design networks.

Figure 13. Three intertwined cycles

\section{Acknowledgments}

The research reported in this paper was carried out as part of the Design Synthesis and Shape Generation (DSSG) project, led by Prof. Alison McKay, University of Leeds, and funded through the AHRC/EPSRC Designing for the 21st Century programme. The authors would like to thank the participants in the study for their cooperation.

Abelson, H and Sussman, G J (1990) Computation: An introduction to engineering design MIT Artificial Intelligence Memo

Agarwal, M and Cagan, J (1998) A blend of different tastes: the language of coffeemakers, Environment and Planning B: Planning and Design Vol 25 No 2 pp 205-226

Bilda, Z and Demirkan, H (2002) An insight on designers' sketching activities in traditional versus digital media, Design Studies Vol 24 No 1 pp 27-50

Cross, N (2006) Designerly ways of knowing Springer, London, UK

Do, E Y-L, Gross, M D, Neiman, B and Zimring, C (2000) Intentions in and relations among design drawings, Design Studies Vol 21 No 5 pp 483-503

Garner, S (1990) Drawing and designing: the case for reappraisal, Journal of Art and Design Education Vol 9 No 1 pp 39-54

Goel, V (1995) Sketches of thought MIT Press, Cambridge, MA

Gross, M D and Do, E Y (1996) Ambiguous intentions: A paper-like interface for creative design, Proceedings of the 9th Annual ACM Symposium on User Interface Software and Technology, Seattle, WA, pp 183 - 192

Knuth, D (1973) Computer science and mathematics, American Scientist Vol 61 No 6 pp 707-13

Koning, $\mathbf{H}$ and Eizenberg, $\mathbf{J}$ (1981) The language of the prairie: Frank Lloyd Wright prairie houses, Environment and Planning B: Planning and Design Vol 8 No 3 pp 295-323

Lawson, B (2006) How designers think (4 ${ }^{\text {th }}$ edition) Oxford: Architectural Press, London, UK

Leclercq, P (1999) Interpretative tool for architectural sketches, in Gero, J S and Tversky, B (eds) Visual and spatial reasoning in design, Key Centre of Design Computing and Cognition, Sydney, Australia, pp 6980

Lim, S, Prats, M, Chase, S, and Garner, S (2008) Categorisation of designs according to preference values for shape rules, in Gero, J S and Goel, A (eds) Design Computing and Cognition '08 (Springer), pp 41-60

Liu, Yu-Tung (1995) Some phenomena of seeing shapes in design, Design Studies Vol 16 No 3 pp 367385

Lloyd, P and Snelders, D (2003) What was Philippe Starck thinking of?, Design Studies Vol 24 No 3 pp 
McKay, A, Jowers, I, Chau, H H, De Pennington, A, and Hogg, D C (2008) Computer aided design: an early shape synthesis system, in Yan, X-T, Ion, W J, Eynard, B (eds) Global Design to Gain a Competitive Edge (Springer, London), pp 3-12

Prats, M, Earl, C, Garner, S, and Jowers, I (2006) Shape exploration of designs in a style: Towards generation of product designs, Al EDAM, Special Issue on Understanding, Representing and Reasoning about Style Vol 20 No 3 pp 201-215

Prats, M and Garner, S (2006) Observations on Ambiguity in Design Sketches, Tracey, an internet-based drawing research journal, Loughborough University

Purcell, A T and Gero, J S (1998) Drawings and the design process, Design Studies Vol 19 pp 389-430

Saund, E and Moran, T P (1994) A perceptually-supported sketch editor, Proceedings of ACM Symposium on User Interface Software and Technology, Marina del Rey, CA, pp 175-184

Schön, D A and Wiggins, G (1992) Kinds of seeing and their functions in designing, Design Studies Vol 13 No 2 pp 135-156

Scrivener, S A R (1982) The interactive manipulation of unstructured images, International Journal of Man-Machine Studies Vol 16 pp 301-313

Stiny, G and Gips, J (1972) Shape grammars and the generative specification of painting and sculpture, in Freiman, C V (ed) Information Processing 71 (Amsterdam: North-Holland), pp 1460-1465

Stiny, G (1990) What is a design?, Environment and Planning B: Planning and Design Vol 17 pp 97-103

Stiny, G (2006) Shape: Talking about seeing and doing, MIT Press, Cambridge, MA

Suwa, M and Tversky, B (1997) What do architects and students perceive in their design sketches?: A protocol analysis, Design Studies Vol 18 No 4 pp 385-403

Van Sommers, P (1984) Drawing and cognition, Cambridge University Press, Cambridge, UK

Woodbury, R F, Burrow, A L (2006) Whither Design Space" Artificial Intelligence for Engineering Design, Analysis and Manufacture, Vol 20 No 2 pp 63-82 


\section{Transforming shape in design: Observations from studies of sketching}

Miquel Prats, The Design Group, The Open University, Milton Keynes, MK7 6AA, UK

Sungwoo Lim, Civil and Building Engineering, Loughborough University, Leicestershire, LE11 3TU, UK lestyn Jowers, Department of Mechanical Engineering, University of Leeds, Leeds, LS2 9JT, UK

Steve Garner, The Design Group, The Open University, Milton Keynes, MK7 6AA, UK

Scott Chase, Department of Design, Manufacture \& Engineering Management, University of Strathclyde, Glasgow, G1 1XJ, UK 


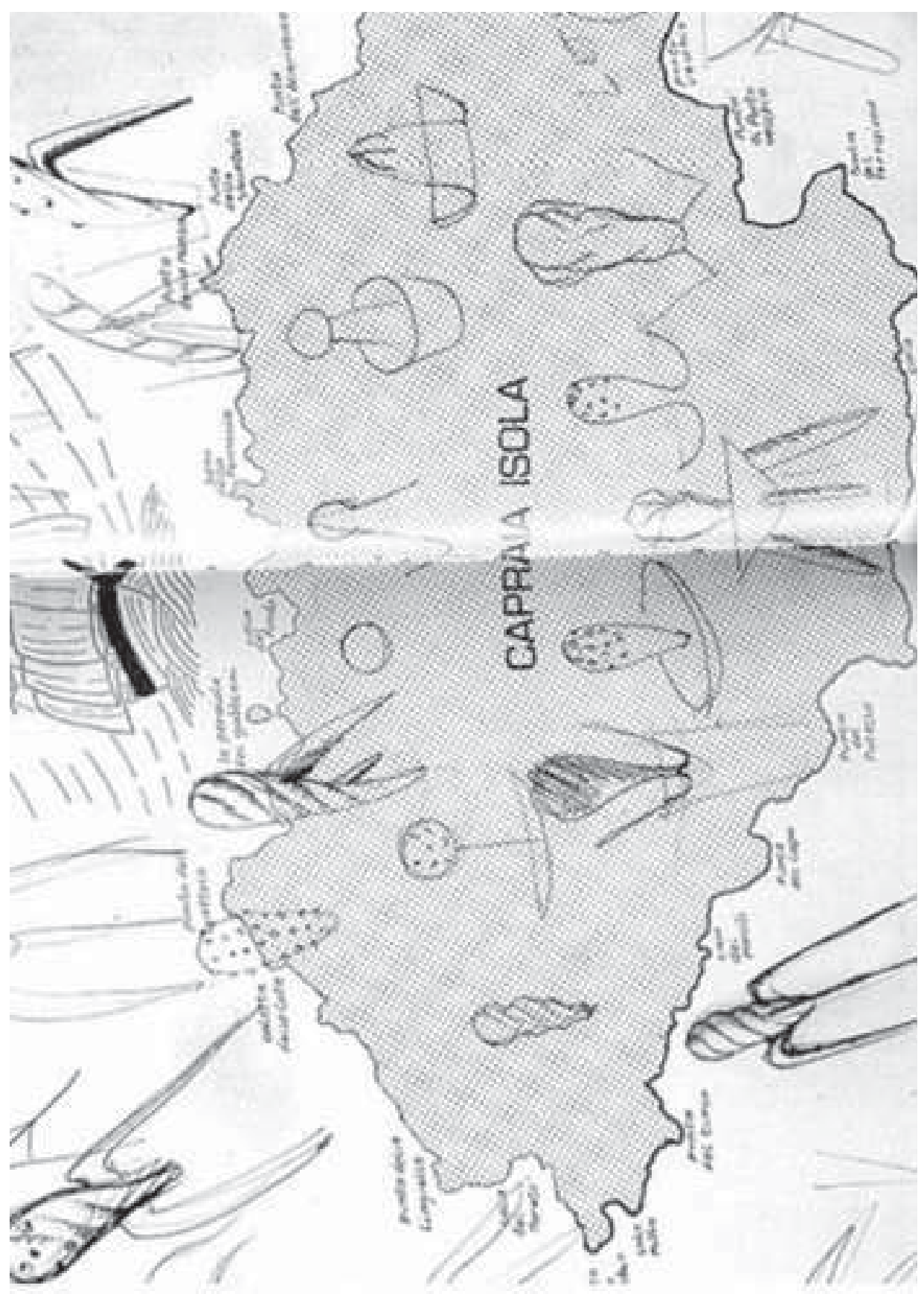

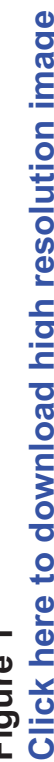

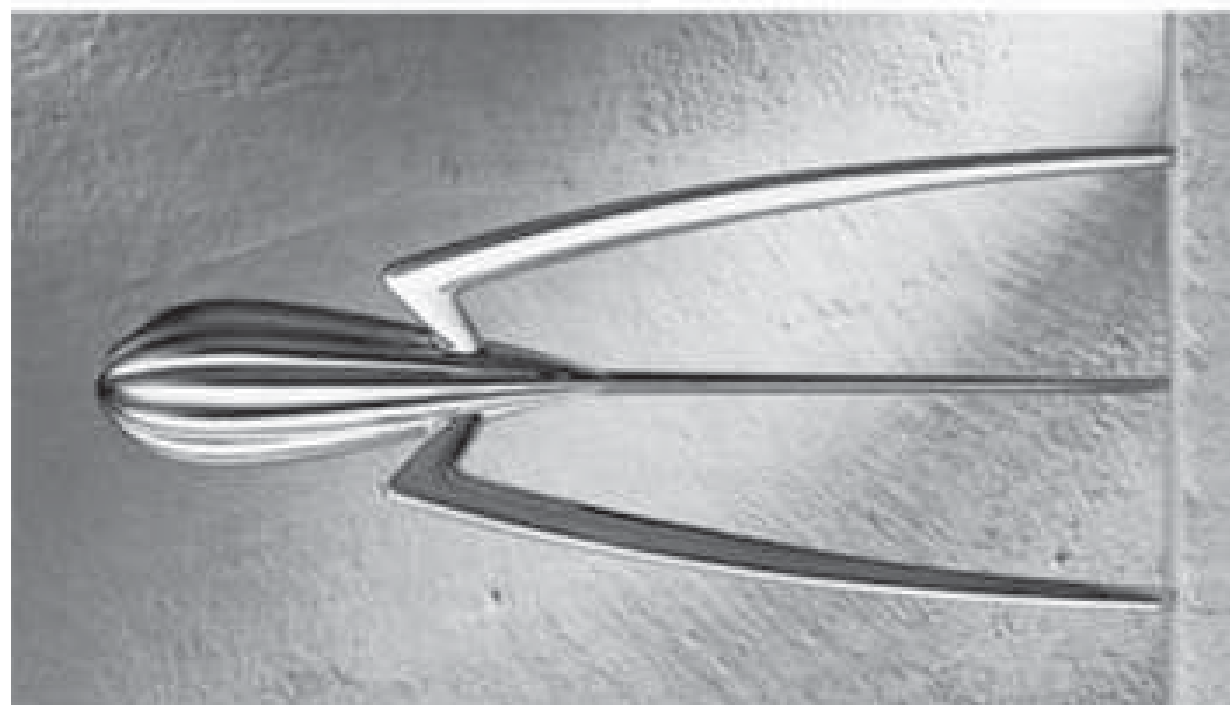



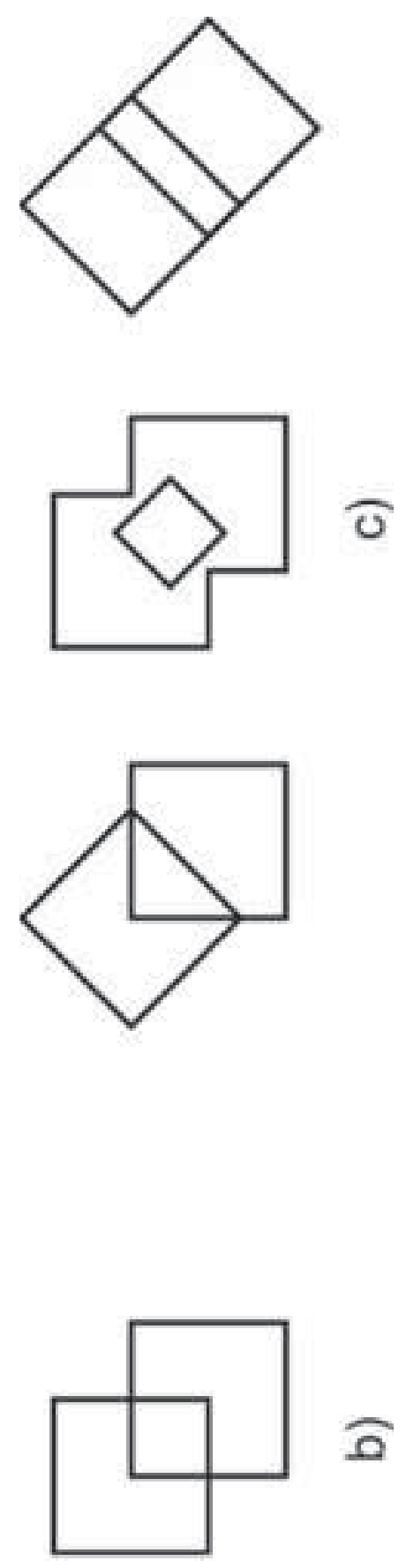

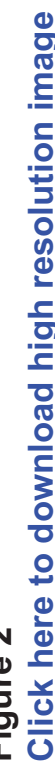

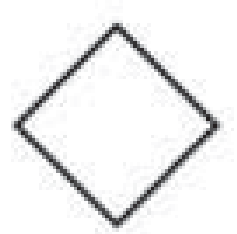

$\uparrow \quad \overparen{\pi}$

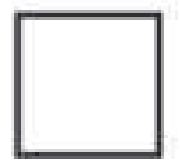



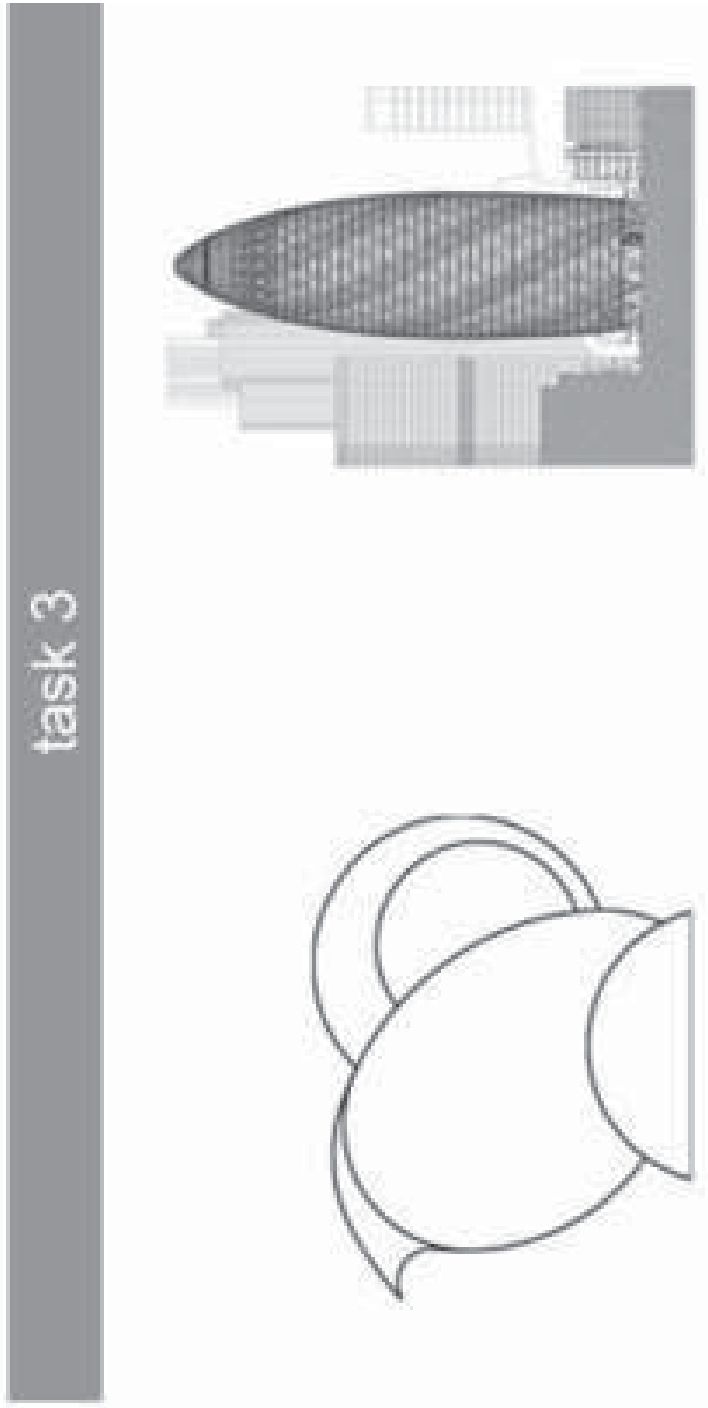

$\frac{\frac{0}{0}}{\frac{d}{}}$

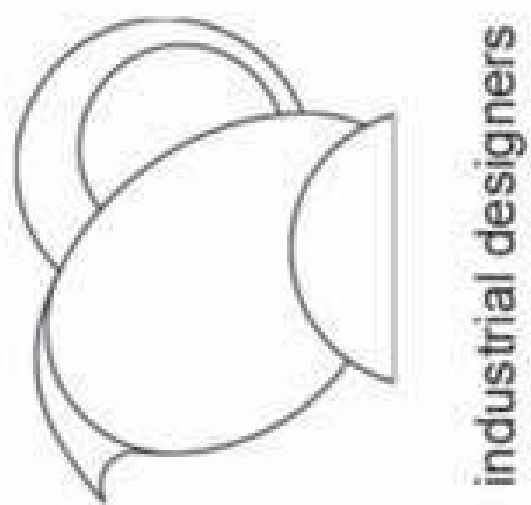

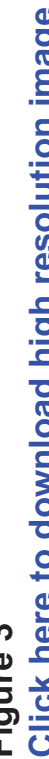
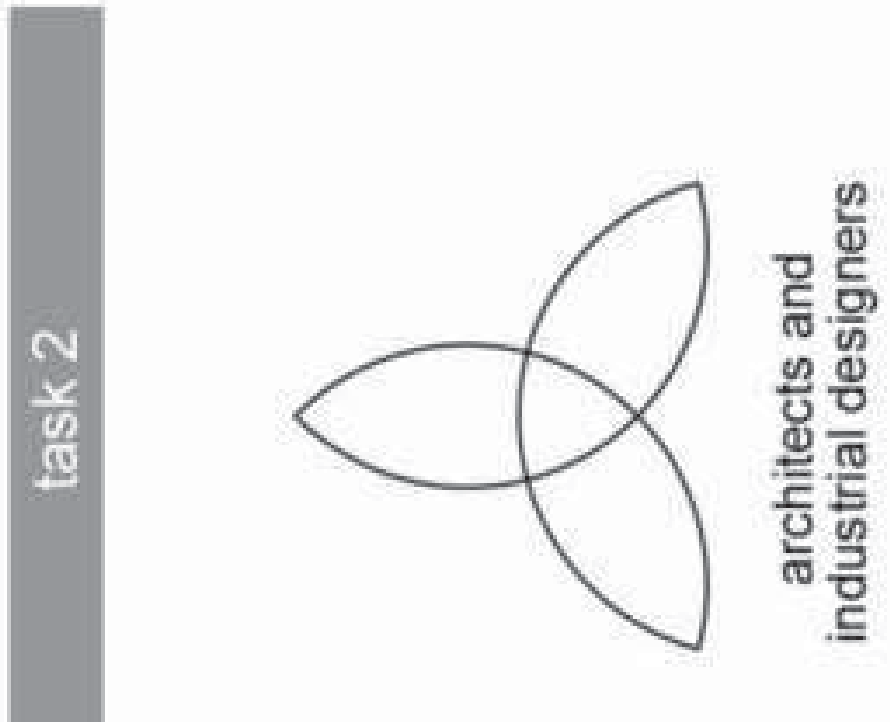


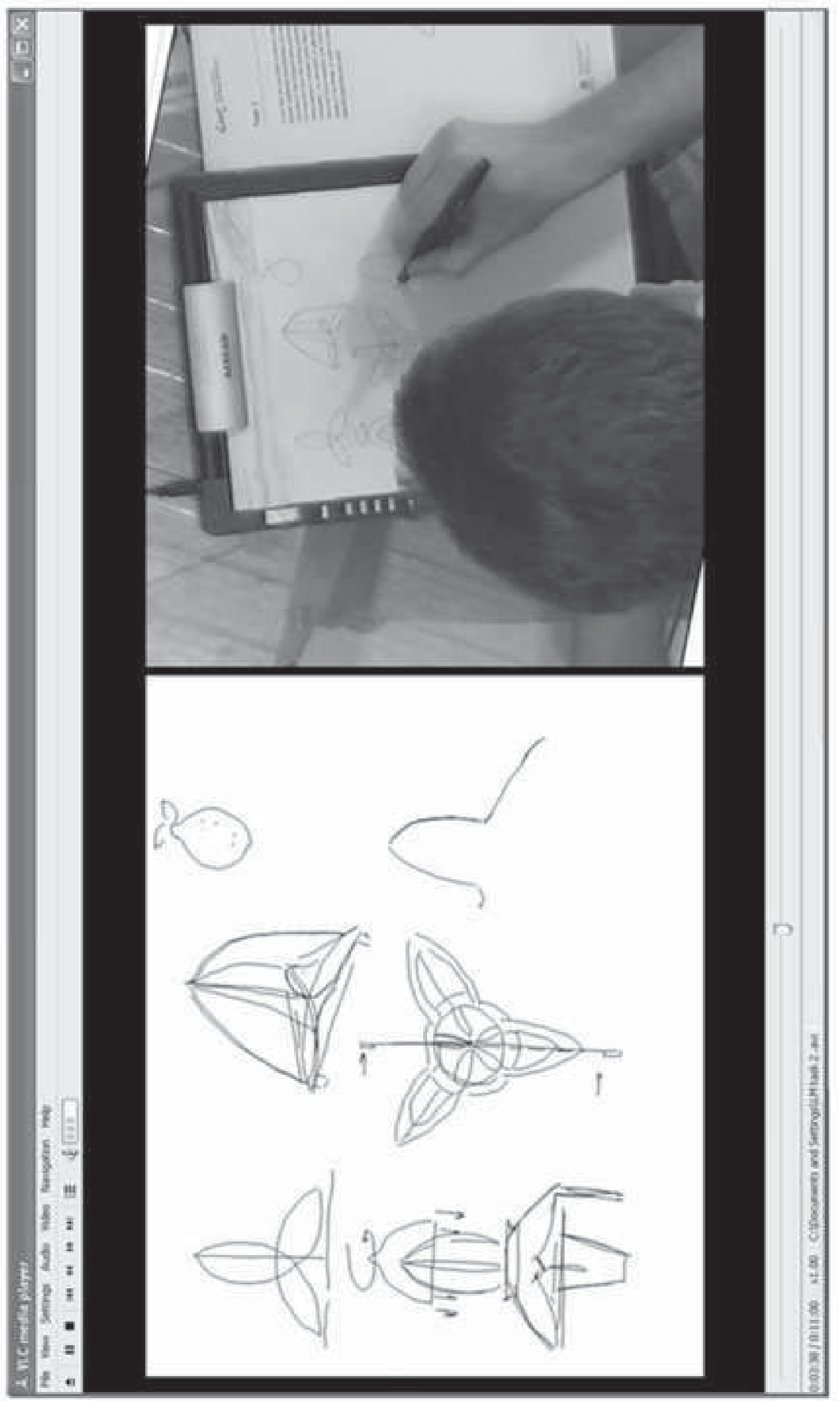




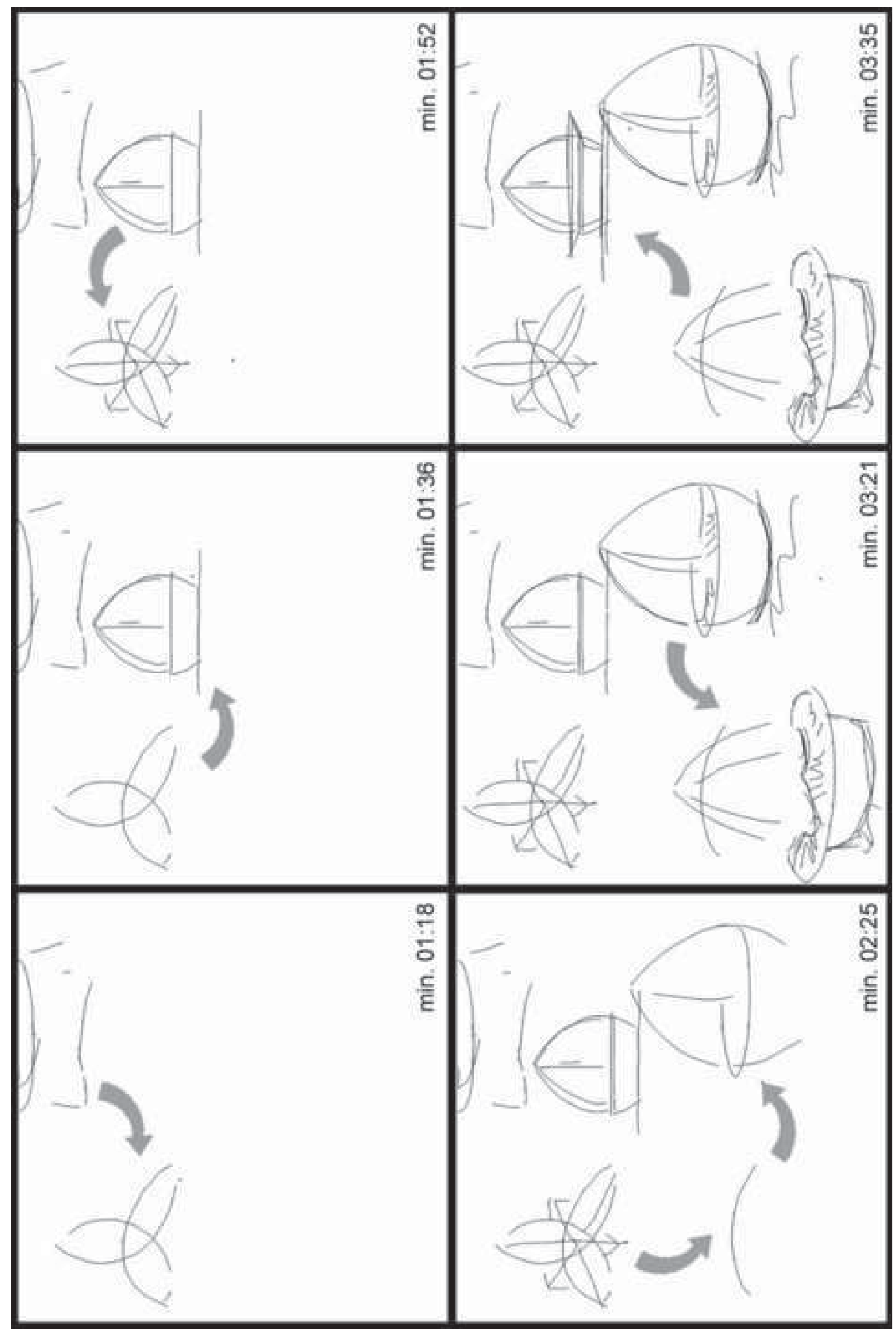



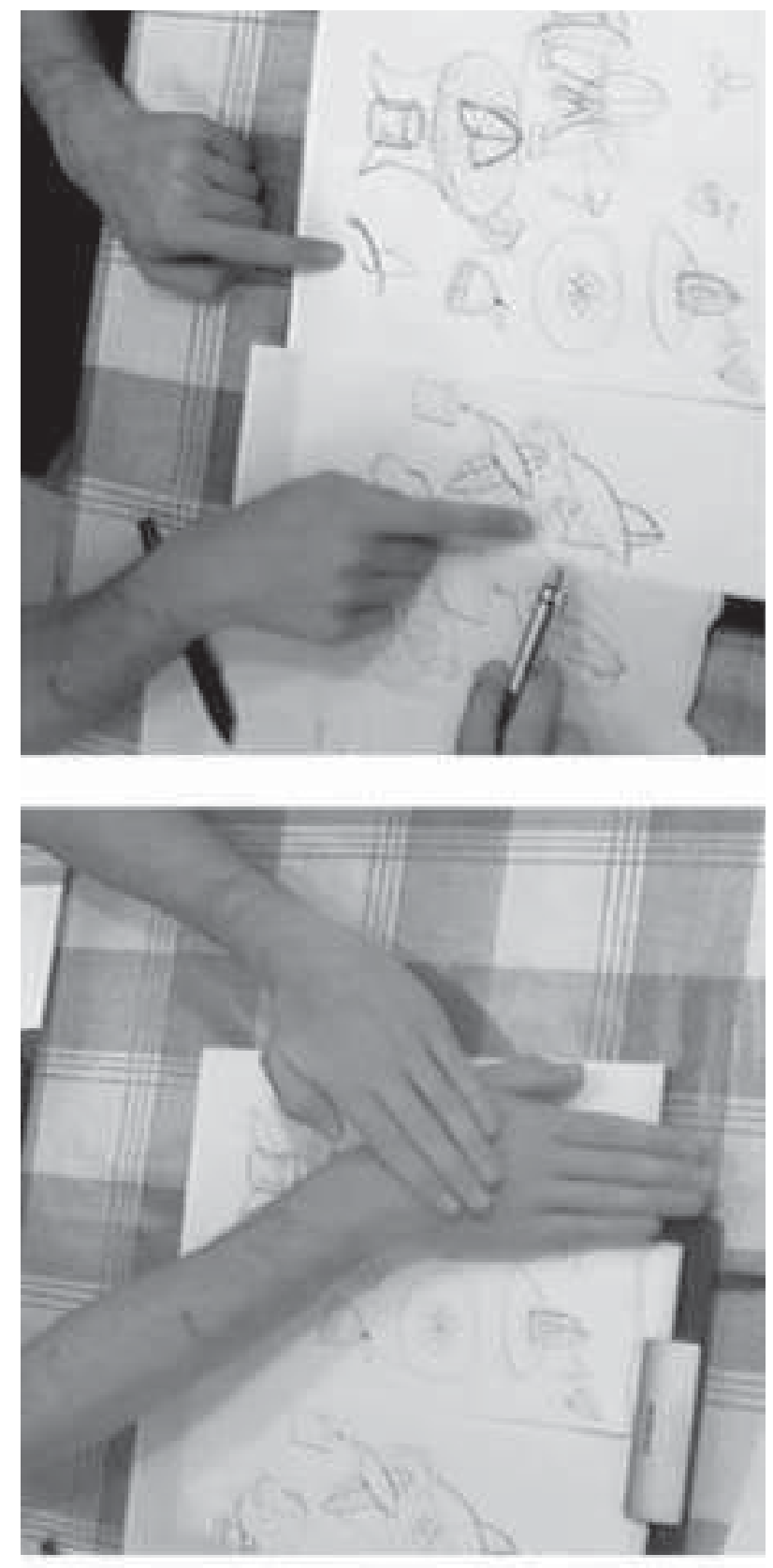

0
0
0

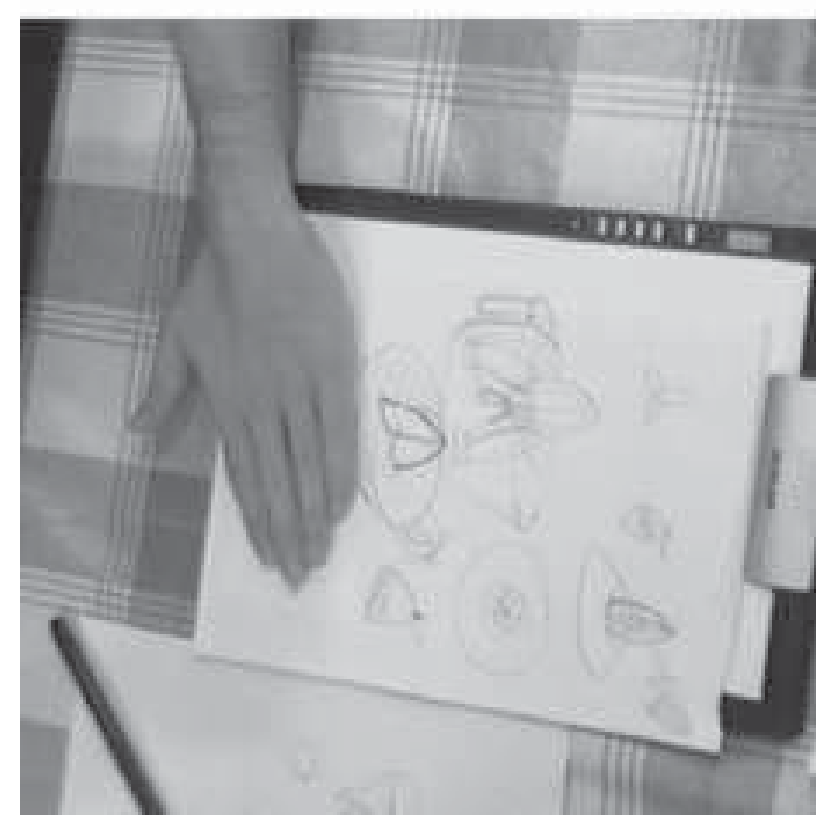




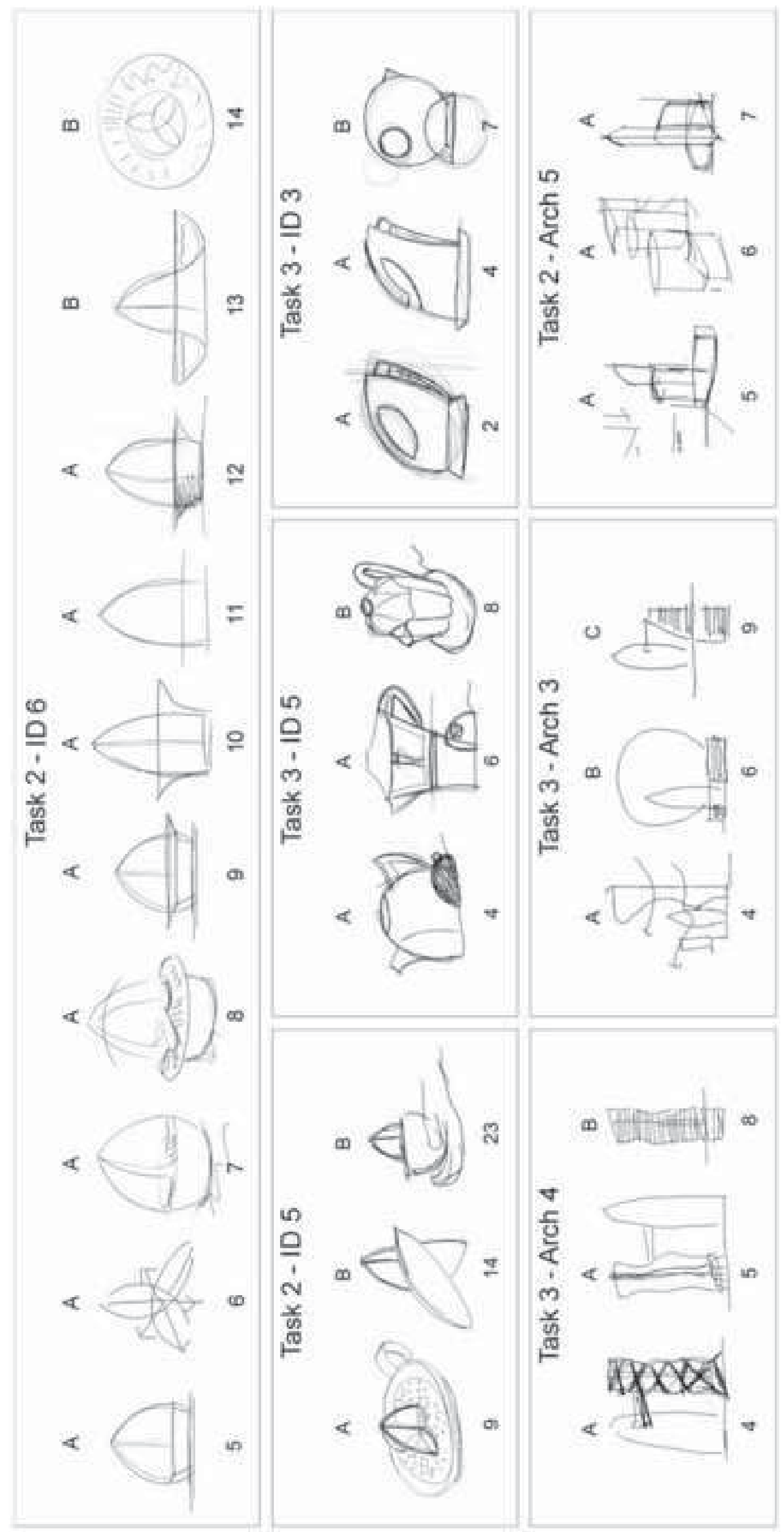

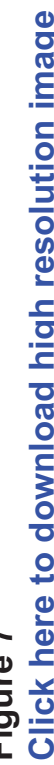


$\uparrow \stackrel{\infty}{\frac{\infty}{2}}$

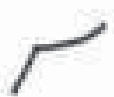

1
$\uparrow \frac{\Phi}{2}$

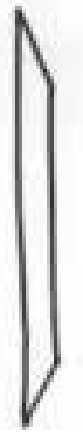

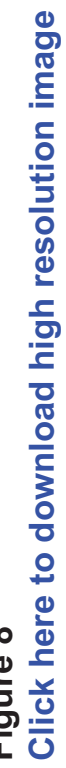

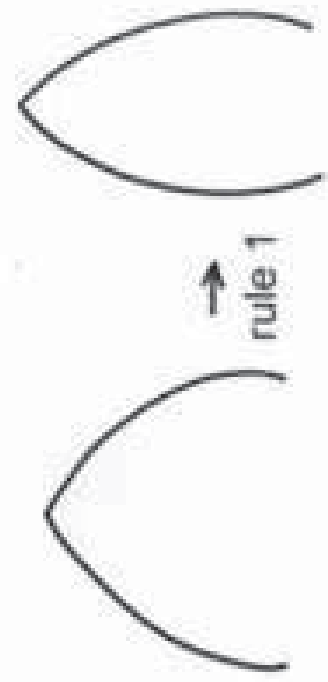




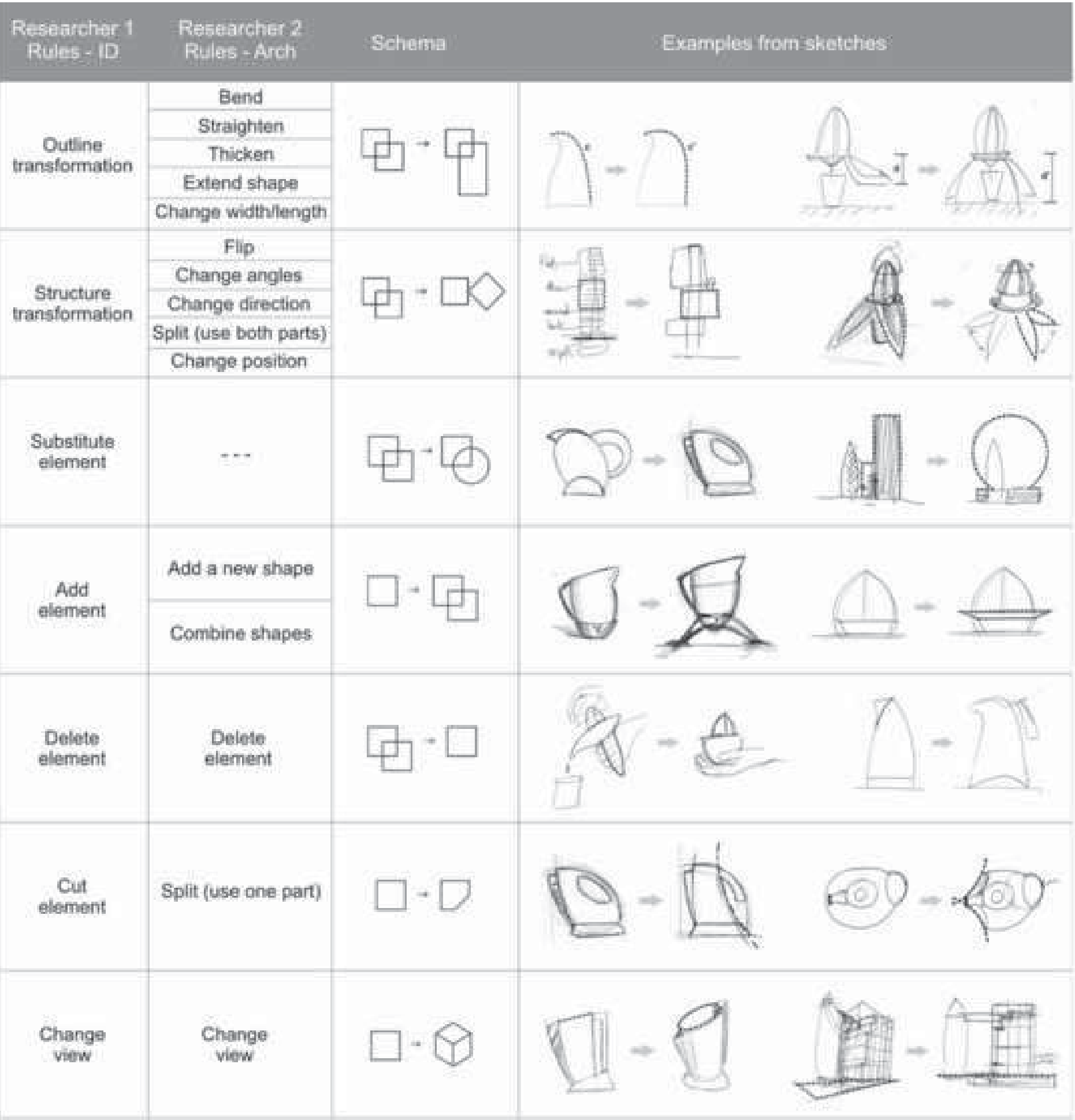


of

of:

of 

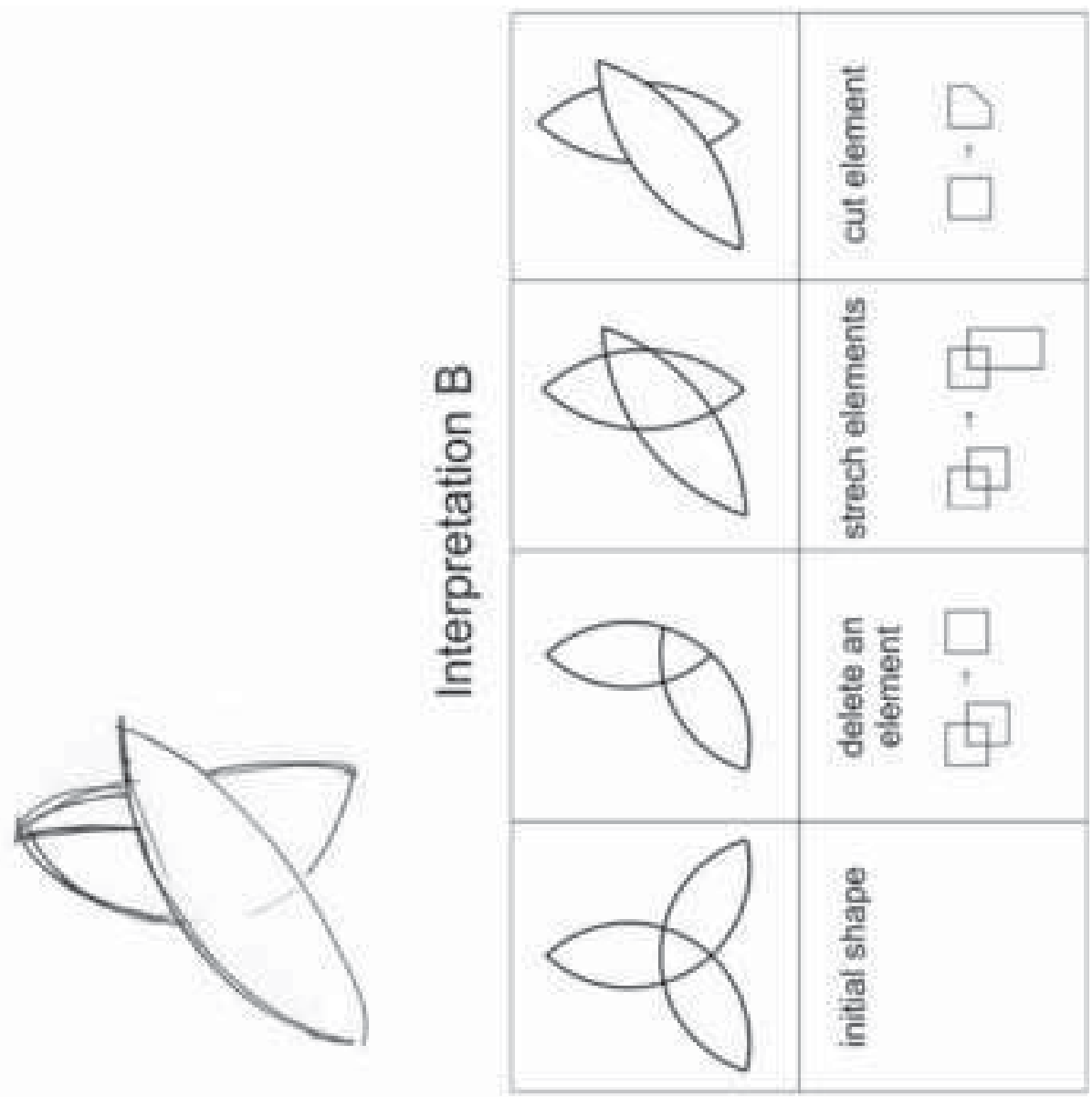

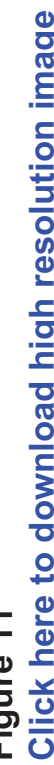

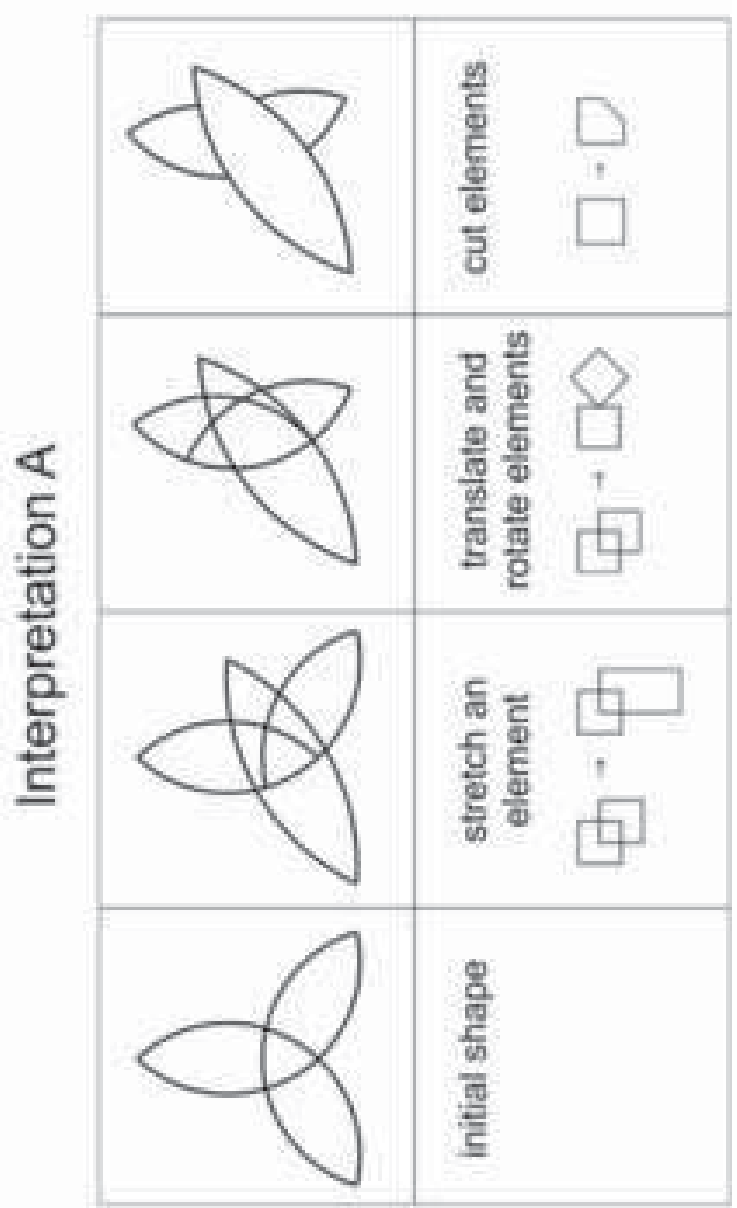




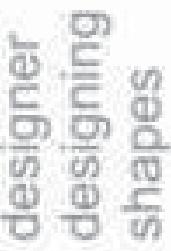
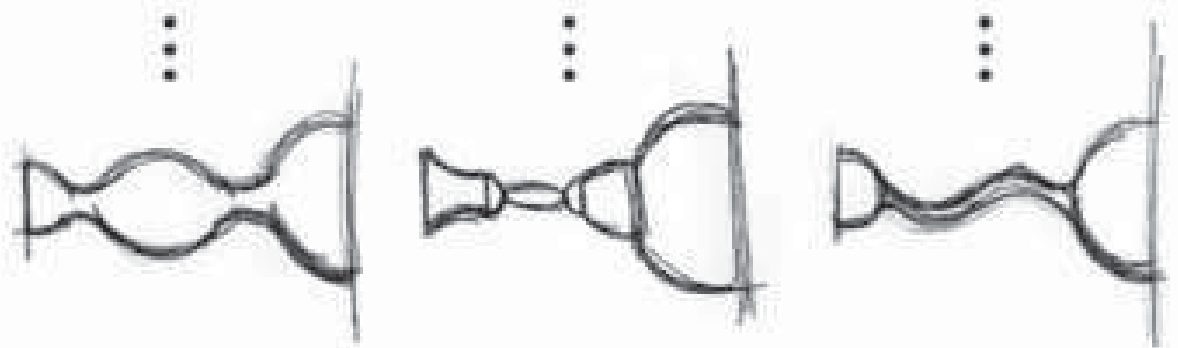

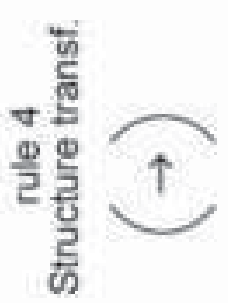

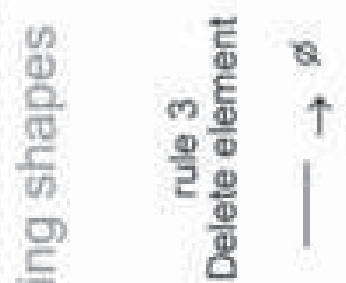

产

穹

总

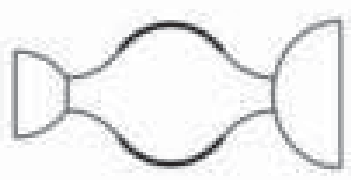

畀的

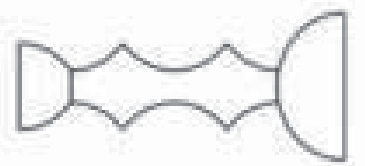

m

$\frac{9}{2}$

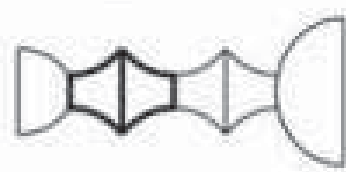

N

黑

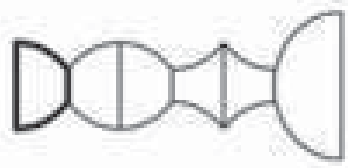

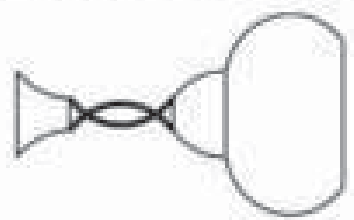

할

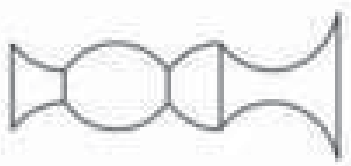

(n)

을

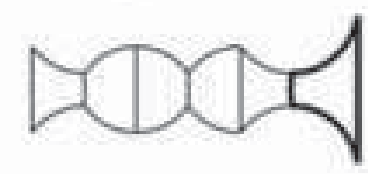

을

$\frac{4}{2}$
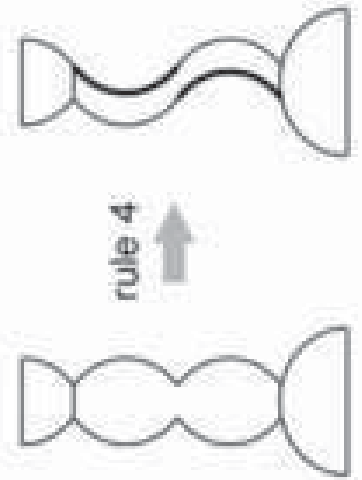

N

을

DODO
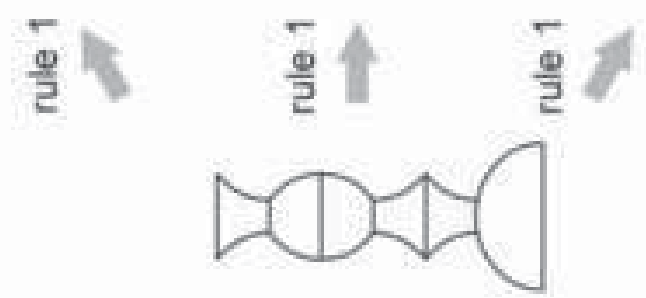

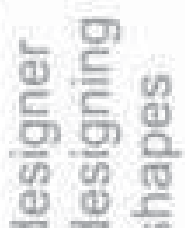
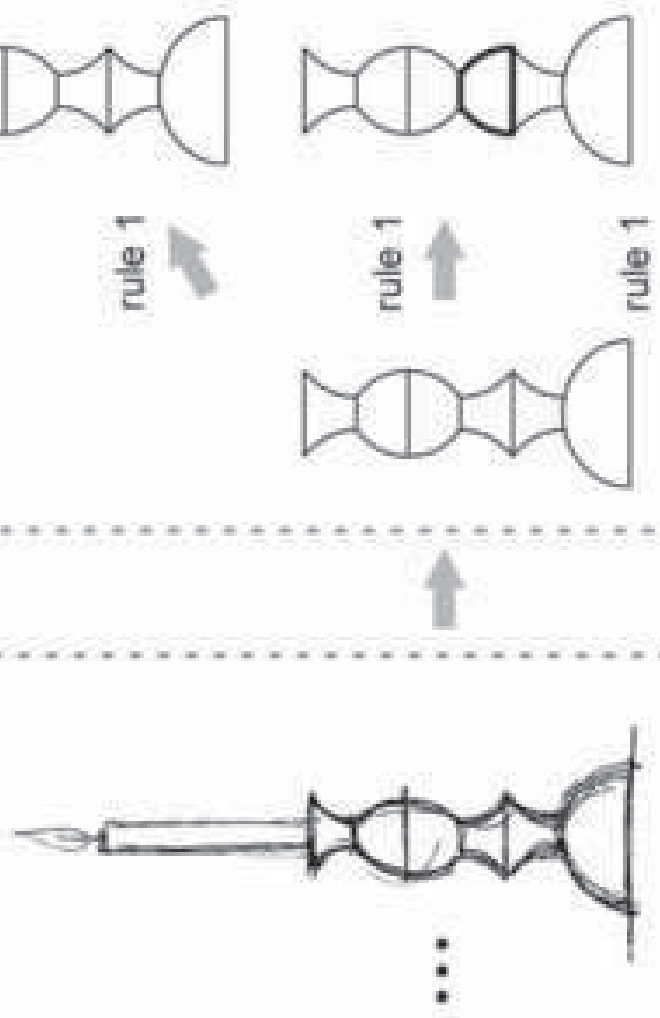


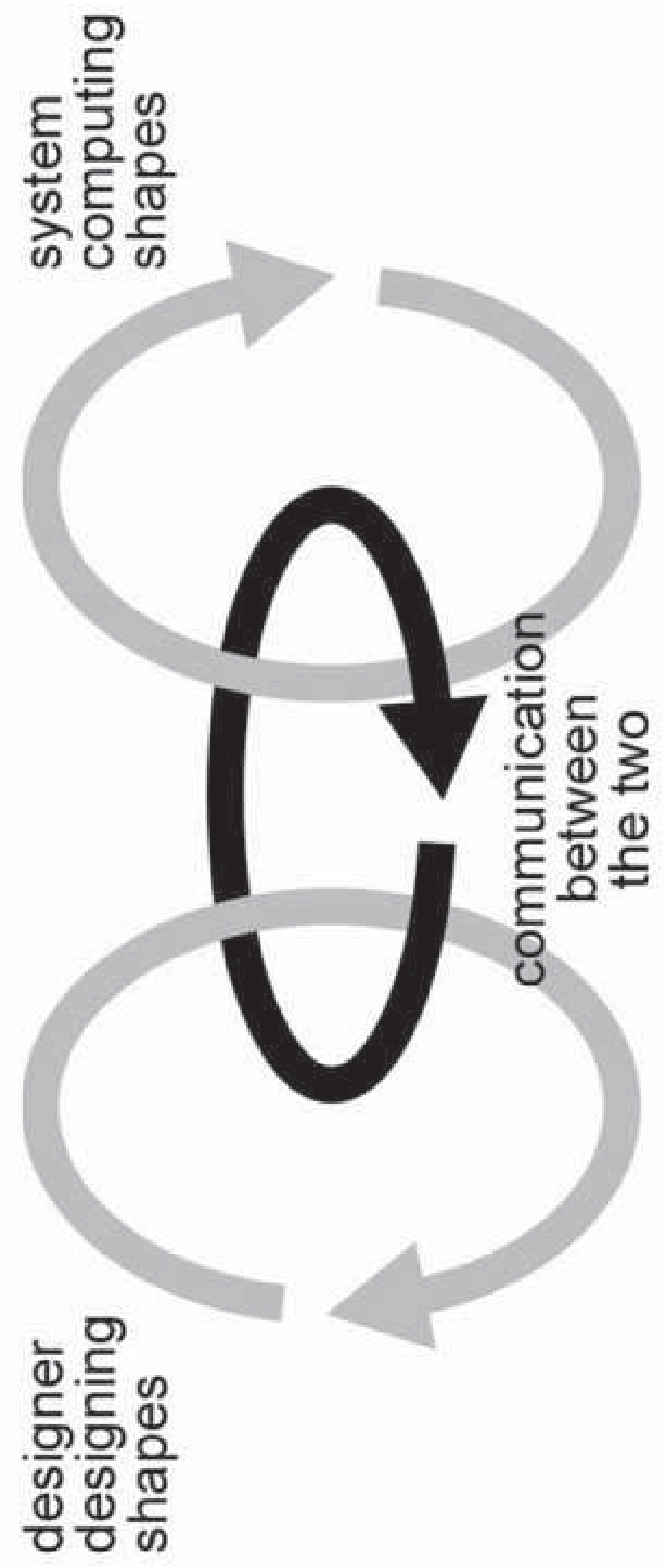

